\title{
Percepción de aceptación y rechazo parental de los hijos y su relación con las características de los padres
}

\section{Ruth Clavijo $^{1}$ (D), María Dolores Palacios ${ }^{1}$ (D), Catalina Mora ${ }^{2}$ (D) Fernando Villavicencio $^{1}$ (D)}

${ }^{1}$ Facultad de Psicología, Universidad de Cuenca, Cuenca, Ecuador.

${ }^{2}$ Universidad Nacional de Educación, Azogues, Ecuador.

Autora para correspondencia: ruth.clavijo@ucuenca.edu.ec

Fecha de recepción:16 de octubre 2017 - Fecha de aceptación: 12 de junio 2018

\section{RESUMEN}

Se investigó la percepción de las manifestaciones de afecto y rechazo parental percibido en niños de 9 a 14 años, prestando atención a variables vinculadas con características de los padres como edad, sexo, tipo de familia y nivel de instrucción. Se trabajó con un muestreo polietápico no probabilístico con la selección de 15 instituciones educativas, de la ciudad de Cuenca; 863 niños, 431 hombres y 432 mujeres con una media de edad de 10.67 años (DT=0.707). Se utilizó la escala Parent PARQ/control Child, versión corta (24 ítems) que incluye afirmaciones sobre el comportamiento de los padres/madres con su hijo; está conformada por cuatro subescalas: cariño / afecto, hostilidad / agresión, indiferenciada / negligencia y rechazo indiferenciado. Se encontró que los niños perciben a sus padres como altamente afectivos con porcentajes por encima de la media, evidenciando ligeras diferencias hacia la madre, quien obtiene mayores puntuaciones en las expresiones de afecto. Si bien el grupo de estudio perciben manifestaciones de afecto y rechazo similares, en las expresiones de indiferencia, agresión y rechazo se exhiben diferencias en función de la edad, el nivel de instrucción de los padres y el tipo de familia.

Palabras clave: Niños, percepción de afecto, rechazo parental.

\begin{abstract}
The parental acceptance-rejection perceived by 9 to 14-year-old children was investigated taking into consideration the parents' characteristics such as age, sex, family type and level of parental education. A non-probabilistic multistage population was selected from 15 educational institutes in CuencaEcuador. Participants were 863 children, 431 male, and 432 female, and the average age was 10.67 years (DT=0.707). The Parental Acceptance-Rejection/Control Questionnaire-Short Form (PARQ/Control-SF), with 24 items, was used to assess parents' behavior with their child about perceived warmth / affection, hostility / aggression, indifference / neglect, and undifferentiated rejection. Results showed that children perceived their parents as highly affective with an above average score, showing a slightly higher score for the mothers. Although participants perceived a similar tendency of parental affection and rejection, differences in the recognized parental attitude of indifference in general, aggression, and rejection were related to parents' age, level of instruction and family type.
\end{abstract}

Keywords: Children, perception of affection, parental rejection.

\section{INTRODUCCIÓN}

Desde la sociología, la psicología y la antropología, se ha situado a la familia como fuente esencial del proceso histórico y construcción social del sujeto (Triana, Ávila, \& Malagón, 2010), representa uno de los núcleos determinantes en el desarrollo cognitivo, personal, emocional y socio afectivo del niño 
(Samper, Cortes, Mestre, Nacher, \& Tur, 2006). En todas las sociedades por muchos años los profesionales de estas áreas proveen descripciones sobre cómo se cría a los niños con marcadas diferencias entre las distintas culturas alrededor del mundo (Del Barrio, Ramírez-Uclés, Romero, \& Carrasco, 2014; Etxebarria, Apodaca, Fuentes, López, \& Ortiz, 2009; Evans \& Myers, 1994). Los niños crecen en una amplia variedad de circunstancias físicas, sociales y culturales, pero la familia es el agente de socialización primario y la institución por excelencia que determina la comunicación, las relaciones afectivas, la organización en la realización de actividades, la importancia de los valores, en definitiva, el clima familiar (Samper et al., 2006). Concretamente son los padres los responsables de las expresiones de afecto o rechazo que despliegan en el cuidado de los hijos, constituyéndose en los agentes más importantes para el desarrollo y bienestar emocional de los niños (Rodríguez, 2017; Samper et al., 2006) con repercusiones desde la niñez hasta la edad adulta (Del Barrio et al., 2014).

La familia en décadas recientes ha pasado por procesos de evolución, donde ha combinado cambio y continuidad para adaptarse a las modificaciones sociales, económicas y tecnológicas (Rodríguez, Del Barrio, \& Carrasco, 2009). Diferentes transformaciones de la sociedad inciden en la diversificación de la estructura familiar, reducción del número de hijos, incremento del número de divorcios, modificaciones de relaciones entre sus miembros y roles que desempeñan (Oudhof van Barneveld, Rodríguez, \& Robles, 2012). Durante mucho tiempo la crianza y sus manifestaciones fue vista como una actividad unilateral de padres a hijos (Oudhof van Barneveld et al., 2012). En la actualidad se reconoce que la familia no es una isla, es un sistema interrelacionado donde las expresiones de afecto y rechazo se determinan no solo por el accionar de los padres (Rodríguez et al., 2009) sino que las relaciones de los padres con los hijos son bidireccionales (Espinal, Gimeno, \& González, 2004). De acuerdo al modelo sistémico la conducta parental no es lineal, por el contrario, las expresiones de aceptación y rechazo de los padres hacia los hijos responde a un proceso de intercambios que presumen una influencia recíproca, bidireccional y circular (Espinal et al., 2004).

La competencia parental está conformada por las conductas del padre y la madre y por el reporte de los hijos sobre esas conductas. Las relaciones entre padres e hijos y la calidad de las mismas presentan diferencias en la percepción que se tenga de la conducta parental, ya sea desde la apreciación de los padres o a partir de la valoración de los hijos (Rohner \& Carrasco, 2014; Torres, Ortega, Garrido, \& Luna, 2008). Según investigaciones revisadas se puede establecer que de acuerdo con el entramado de relaciones paternofiliales se extraen diferentes formas de actuar y de comportamiento de los padres en la interacción con sus hijos, ello influye directamente en su conducta, en las formas de actuar y en las relaciones con su entorno (Del Barrio et al., 2014; Etxebarria et al., 2009; Polo del Río, Fajardo, Martín, Gómez, \& León, 2012; Rodríguez, 2017).

La conducta parental en todas las sociedades humanas se manifiesta por medio de dos grandes dimensiones: la aceptación y el rechazo (Gracia, Lila, \& Musitu, 2005), que se expresan en un continuo caracterizado por padres que en un extremo demuestran amor, aceptación y afecto, y en otro extremo, rechazo, trato severo y abusivo hacia sus hijos. El rechazo parental puede adoptar formas diversas como la agresividad y la hostilidad, que desencadenan estilos parentales basados en control físico y verbal o la indiferencia (Richaud de Minzi, Lemos, \& Mesurado, 2011). La aceptación parental percibida (calidad parental) por los hijos se ha mostrado como el mecanismo a través del cual la implicación del padre y la madre contribuye al ajuste de la personalidad de los niños e inhibe sus problemas de comportamiento (Samper et al., 2006). La implicación de los padres afecta a los resultados de los niños no solo cuando éstos perciben una relación paterna positiva, sino cuando se evidencia percepción de manifestación de agresión y rechazo (Del Barrio et al., 2014; Rodríguez, 2017).

Numerosos autores señalan la necesidad de conocer la percepción de los hijos sobre los hábitos de crianza y las expresiones de afecto y rechazo de sus padres (Etxebarria et al., 2009; Oudhof van Barneveld et al., 2012; Richaud de Minzi et al., 2011; Rodríguez, 2017). En algunos estudios se analiza el rol de la autoridad familiar, el afecto y rechazo parental y su incidencia en el comportamiento de niños y niñas en diferentes contextos (Rohner \& Carrasco, 2014). Estos estudios señalan que la información obtenida de los hijos es más confiable que aquella que se obtiene de los padres (Álvarez, 2010; Rodríguez et al., 2009). La información manifestada por los hijos se ha mostrado no sólo fiable sino más coherente que la suministrada por los padres en relación con otras fuentes. Gaylord, Kitzmann, \& Coleman (2003) encontraron que la información proporcionada por los hijos sobre la conducta parental posee mayor validez predictiva sobre el grado de aceptación entre sus iguales, frente a la 
información expresada por sus padres. La razón que puede explicar esta diferencia es que la información suministrada por los padres parece estar sometida a una mayor deseabilidad social (Rodríguez et al., 2009). Al respecto Rodríguez (2017) indica que la implicación parental es crucial para el desarrollo cognitivo, conductual, psicológico, emocional y relacional de los niños, tal como sostienen otros estudios (Caycho, 2016; Rodríguez et al., 2009; Rohner \& Carrasco, 2014).

Un estudio sobre "Aceptación-rechazo de padres y madres cuencanos relacionados a las características personales de los hijos entre 5 y 12 años" reportó que 595 padres/madres de niños entre los 5 y 12 años de escuelas de la ciudad de Cuenca, tienen una inclinación positiva hacia el afecto. Los padres se consideran afectivos, así lo registró el 95.5\% de los participantes (Clavijo et al., 2017); estos resultados obtenidos permiten estimar que los padres son mayoritariamente afectuosos con los hijos.

Desde la IPARTheory ${ }^{1}$, teoría de la aceptación-rechazo interpersonal se valora las dimensiones de la socialización familiar enfocadas a la aceptación y rechazo parental, como dos aspectos importantes para evaluar la incidencia que tienen las pautas educativas parentales, en el desarrollo de los niños (Del Barrio et al., 2014; Rohner \& Carrasco, 2014). Las prácticas parentales se definen como aquellas conductas que los padres utilizan para socializar a sus hijos. Las caracterizadas como frías, hostiles, poco afectuosas, agresivas, indiferentes o negligentes, consistentemente se asocian con consecuencias negativas en el comportamiento de los hijos. Por el contrario, cuando el comportamiento parental se define por ser afectuoso, cariñoso, democrático y emocionalmente cálido, las consecuencias en los hijos son positivas (Rodríguez, 2017). De acuerdo con la IPARTheory, la percepción por parte de los niños de que sus padres los aceptan, respetan sus opiniones, están orgullosos de ellos, aumenta su seguridad y les permite establecer relaciones positivas y altruistas con los demás (Richaud de Minzi et al., 2011; Rohner \& Carrasco, 2014). Según estos autores, la percepción sobre manifestaciones de rechazo, indiferencia o negligencia se asocian con sentimientos de incapacidad, aislamiento, conducta antisocial y sentimientos de inferioridad.

Existe un consenso general sobre el hecho de que las interacciones tempranas entre los niños y sus principales cuidadores constituyen una de las influencias formativas más relevantes en el desarrollo posterior de niños y niñas, en particular debido a que la evidencia teórica sugiere una estrecha relación entre la calidad de las interacciones padres-hijos y la temporalización en la consecución de los hitos del desarrollo (Rodríguez et al., 2009; Rodríguez, 2017). Rodríguez et al. (2009) sugieren que la interacción directa con el niño, la disponibilidad, o la responsabilidad en su cuidado se asocia con resultados positivos, calidad de crianza, sobre todo una relación afectuosa entre padres e hijos. Con el sustento teórico señalado, el presente trabajo tuvo por objetivo identificar la percepción de aceptación y rechazo parental de los hijos entre nueve y catorce en años con relación a las características de los padres, en cuanto a la edad, sexo, tipo de familia y nivel de instrucción.

\section{MATERIALES Y MÉTODO}

\subsection{Población y muestra}

Se accedió a los informantes a través de un muestreo polietápico no probabilístico: se seleccionaron 15 instituciones educativas de la ciudad de Cuenca que ofertan Educación General Básica (EGB) (9 fiscales y 6 particulares), de las cuales por conveniencia se seleccionaron 863 niños, $49.9 \%$ hombres $(n=431)$ y $50.1 \%$ mujeres $(n=432)$ de entre 9 y 14 años, con una media de edad de 10.67 años (DT=0.707).

\subsection{Características de la muestra}

El $97.2 \%$ de los participantes respondieron que su madre era la figura cuidadora, el 1.9\% su abuela y el $0.9 \%$ eran cuidados por su tía, hermano o madrastra. El 52\% reportó vivir en una familia nuclear, el $21 \%$ en familias monoparentales maternas, el $20 \%$ en familias extendidas, el $6 \%$ en familias reconstituidas y el $1 \%$ en familias monoparentales paternas. El $36.6 \%$ de niños consultados señalaron tener un hermano, el $33.9 \%$ dos hermanos, el $22.4 \%$ tres o más hermanos y el $6.8 \%$ hijos únicos. En

${ }^{1}$ IPARTheory: Interpersonal acceptance-rejection theory (Rohner \& Carrasco, 2014) 
general, los progenitores registraron edades entre 24 y 81 años, con una edad media de 38.95 años ( $\mathrm{DE}=6.87$ ). Los padres reportaron edades de entre 28 y 81 años, con una media de edad de 40.45 años $(\mathrm{DE}=7.4)$; el $76.6 \%$ de ellos casados, seguido de un 7.3\% divorciados. Respecto al nivel de instrucción se destaca el $39.3 \%$ con instrucción básica y el $43.7 \%$ con bachillerato (incluyen aquellos con nivel superior incompleto). Las madres reportaron edades entre 24 y 55 años, con una media de edad de 37.39 años (DE=5.99); el $70.9 \%$ casadas, seguido de un $10.3 \%$ divorciadas. En cuanto al nivel de instrucción el $29.9 \%$ señala contar con educación básica, el $32.3 \%$ bachillerato y el $35.1 \%$ educación superior. La Tabla 1 ofrece un resumen de los datos sociodemográficos de los progenitores.

Tabla 1. Datos sociodemográficos de los padres $(n=765)$ y madres $(n=837)$.

\begin{tabular}{lcc}
\hline Situación conyugal & Padres (\%) & Madres (\%) \\
\hline Casado & 76.6 & 70.9 \\
Unión libre & 5.4 & 5.9 \\
Separado & 1.6 & 1.6 \\
Divorciado & 7.3 & 10.3 \\
Viudo & 0 & 0.7 \\
Soltero & 3.9 & 9.6 \\
NC & 5.2 & 1.1 \\
\hline
\end{tabular}

\begin{tabular}{lll}
\hline Instrucción & Padres (\%) & Madres (\%) \\
\hline Ninguno & 0.5 & 0.7 \\
Básica & 26 & 29.9 \\
Bachillerato & 31.7 & 32.3 \\
Superior & 35.2 & 35.1 \\
NC & 6.6 & 2.1 \\
& &
\end{tabular}

NC: No contesta

\subsection{Recolección de datos}

La investigación se desarrolló como un estudio cuantitativo de tipo descriptivo y relacional, para el efecto se empleó la escala "CHILD PARQ/Control"2", debidamente autorizada, a dicha escala se integró en una primera sección información sociodemográfica de los niños consultados y de sus padres y madres. La escala de 24 ítems incluye afirmaciones sobre el comportamiento de padres y madres con su hijo; las respuestas tipo Likert corresponden a la frecuencia con la que esas expresiones son percibidas por su hijo: siempre $=4$, muchas veces $=3$, pocas veces $=2$, nunca $=1$. Está conformada por cuatro tipos de expresiones: cariño / afecto (8 ítems), hostilidad / agresión (6 ítems), indiferencia / negligencia (6 ítems), rechazo indiferenciado (4 ítems). Las cuatro expresiones corresponden a la variable aceptación-rechazo parental. Para la interpretación de los valores asignados se invierten las puntuaciones de la escala cariño/afecto (mide frialdad), se suman los valores asignados a los 24 ítems de la subescala, los puntajes totales oscilan entre 24 a 96 puntos, de esa forma puntajes bajos implican frecuentes manifestaciones de afecto y puntajes altos implican frecuentes manifestaciones de rechazo. De igual forma valores altos en cada una de las cuatro expresiones representan frecuentes manifestaciones de: afecto, indiferencia, agresión y rechazo.

El instrumento reportó un alpha de Cronbach de .869 y la versión padre un alpha de Cronbach de 0.906. La fiabilidad interna de las subescalas: afecto, indiferencia, agresión y rechazo oscila entre .58 y .85 (Tabla 2).

Tabla 2. Coeficiente de fiabilidad subescalas, escala aceptación-rechazo parental.

\begin{tabular}{lcc}
\hline Subescala & Versión madre $^{3}$ & Versión padre $^{3}$ \\
\hline Afecto & 0.83 & 0.85 \\
Indiferencia & 0.76 & 0.76 \\
Agresión & 0.65 & 0.69 \\
Rechazo & 0.58 & 0.65 \\
\hline
\end{tabular}

\footnotetext{
2 Child-PARQ/Control Father/Mother: Child-Parental Acceptance-Rejection Questionnaire (Rohner, 2005)

3 Alpha de Cronbach
} 


\subsection{Procedimiento}

Se gestionó la autorización de la Coordinación Zonal 6 de Educación para el acceso a los informantes, se coordinó y organizó la recolección de información con los directores de 15 instituciones educativas participantes. En sobre cerrado, los niños de quinto a séptimo de básica de los paralelos seleccionados llevaron una carta informativa, la hoja de asentimiento informado y el cuestionario de datos sociodemográficos a ser respondido por los padres, madres o cuidadores. Con los consentimientos de los representantes legales se aplicaron los cuestionarios a los niños, el procedimiento estuvo a cargo de los integrantes del equipo de investigación.

\subsection{Análisis estadístico}

Para el análisis de los datos se utilizó el programa estadístico SPSS versión 23, la distribución asimétrica de los puntajes de la escala de medición determinó el empleo de pruebas de hipótesis no paramétricas: Mann-Withney U test y Kruskall-Wallis $\mathrm{H}$ test. Las decisiones se tomaron con un nivel de significancia de .05 .

\section{RESULTADOS}

El puntaje de la variable afecto-rechazo parental registró una media de 35.93 (DE=10.473) para los padres y una media de 35.56 (DE=9.45) para las madres, la distribución de frecuencia en los dos casos reportó una asimetría positiva de 1.68 (Fig. 1a) y de 1.5 (Fig. 1b), respectivamente, lo cual implica que los hijos perciben aceptación de sus padres y madres.

Figura 1. Distribución de frecuencias de la variable aceptación-rechazo parental.
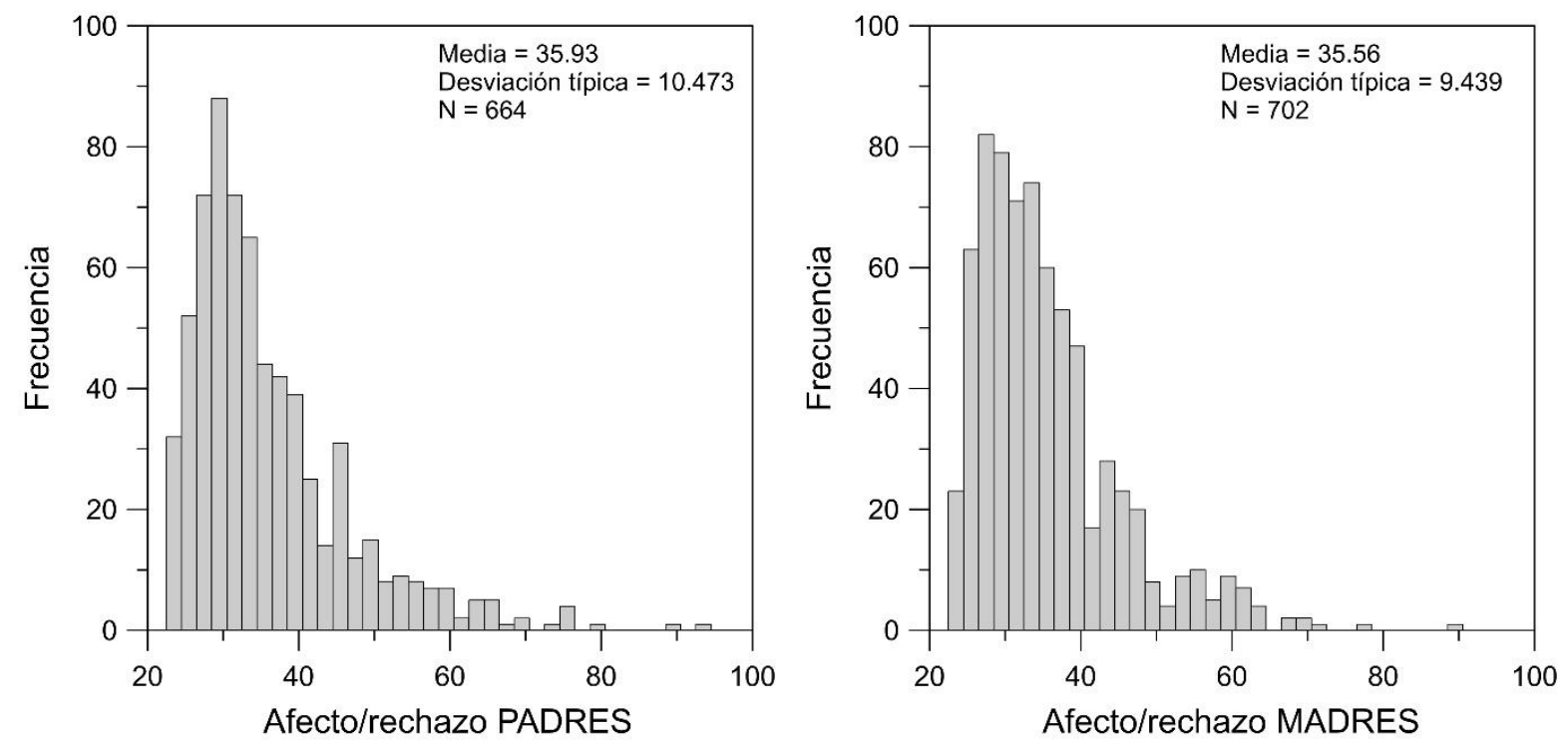

(a) Distribución de frecuencias de la variable aceptación/rechazo Padres.

(b) Distribución de frecuencias de la variable aceptación/rechazo Madres.

Ya que la variable aceptación/rechazo parental involucra expresiones de: afecto, indiferencia, agresión y rechazo, se analizaron las frecuencias de estas expresiones tanto en padres como en madres. Se encontró que los hijos perciben frecuentes expresiones de afecto de sus padres y madres, así como escazas expresiones de indiferencia, agresión y rechazo. Sin embargo, se perciben con mayor frecuencia expresiones de afecto de sus madres $(p=.006)$ así como expresiones de indiferencia, agresión y rechazo $(p<.05)$ (Tabla 3). 
Tabla 3. Afecto, indiferencia, agresión y rechazo parental.

\begin{tabular}{lcccccc}
\hline \multirow{2}{*}{ Expresiones } & \multicolumn{2}{c}{ Padres } & \multicolumn{2}{c}{ Madres } & \multicolumn{2}{c}{ Estadísticos } \\
\cline { 2 - 7 } & $\mathrm{M}$ & $\mathrm{DE}$ & $\mathrm{M}$ & $\mathrm{DE}$ & $\mathrm{U}$ test & $p$ \\
\hline Afecto & 27.7 & 4.5 & 28.3 & 3.9 & 231974.5 & $.006^{* *}$ \\
Indiferencia & 9.7 & 3.4 & 11.5 & 2.4 & 160211.5 & $.000^{* *}$ \\
Agresión & 8.9 & 2.9 & 9.4 & 2.8 & 218425 & $.000^{* *}$ \\
Rechazo & 5 & 1.7 & 5.1 & 1.7 & 243273.5 & $.009^{* *}$ \\
\hline
\end{tabular}

** Diferencia significativa a nivel de .01

Como se muestra en la Tabla 4, se encontraron diferencias significativas en la frecuencia con la que los hijos perciben agresión y rechazo de sus progenitores y el nivel de instrucción de estos últimos. Así, los progenitores que registraron instrucción básica manifiestan con mayor frecuencia expresiones de agresión y rechazo $(p=.001)$.

Tabla 4. Expresiones de aceptación / rechazo parental y nivel de instrucción de los progenitores.

\begin{tabular}{|c|c|c|c|c|c|c|c|c|c|c|}
\hline \multirow{3}{*}{ Expresiones } & \multicolumn{8}{|c|}{ Nivel de Instrucción } & \multirow{2}{*}{\multicolumn{2}{|c|}{ Estadísticos }} \\
\hline & \multicolumn{2}{|c|}{ Ninguno } & \multicolumn{2}{|c|}{ Básica } & \multicolumn{2}{|c|}{ Bachillerato } & \multicolumn{2}{|c|}{ Superior } & & \\
\hline & $\mathrm{M}$ & $\mathrm{DE}$ & $\mathrm{M}$ & $\mathrm{DE}$ & $\mathrm{M}$ & $\mathrm{DE}$ & $\mathrm{M}$ & $\mathrm{DE}$ & $\mathrm{H}$ test & $p$ \\
\hline Afecto & 28.0 & 3.4 & 27.5 & 4.6 & 28.1 & 4.2 & 28.6 & 3.8 & 2.89 & .236 \\
\hline Indiferencia & 10.4 & 3.0 & 10.9 & 3.3 & 10.5 & 3.0 & 10.5 & 3.0 & 1.59 & .450 \\
\hline Agresión & 8.2 & 1.7 & 9.6 & 3.0 & 9.0 & 2.9 & 9.0 & 2.9 & 13.68 & $.001 * *$ \\
\hline Rechazo & 4.4 & 0.7 & 5.4 & 2.0 & 5.0 & 1.6 & 4.9 & 1.6 & 14.219 & $.001 * *$ \\
\hline
\end{tabular}

** Diferencia significativa a nivel de .01

Se comparó la percepción de la frecuencia de las expresiones de afecto, indiferencia, agresión y rechazo y la edad de los progenitores (Tabla 5), y se encontró diferencia significativa en las manifestaciones de rechazo, siendo mayor en los progenitores menores a los 38 años $(p=.033)$, que constituye la edad promedio del grupo de estudio.

Tabla 5. Expresiones de aceptación / rechazo parental y edad de los progenitores.

\begin{tabular}{lcccccc}
\hline \multirow{2}{*}{ Expresiones } & \multicolumn{2}{c}{38 años o menos } & \multicolumn{2}{c}{39 años o más } & \multicolumn{2}{c}{ Estadísticos } \\
\cline { 2 - 7 } & $\mathrm{M}$ & $\mathrm{DE}$ & $\mathrm{M}$ & $\mathrm{DE}$ & $\mathrm{U}$ test & $p$ \\
\hline Afecto & 27.99 & 4.31 & 28.19 & 4 & 237784 & .939 \\
Indiferencia & 10.7 & 3.08 & 10.53 & 3.01 & 236228 & .41 \\
Agresión & 9.28 & 2.98 & 9.03 & 2.84 & 232286 & .163 \\
Rechazo & 5.13 & 1.76 & 4.97 & 1.6 & 231876.5 & $.033^{*}$ \\
\hline
\end{tabular}

* Diferencia significativa a nivel de .05

Considerando el sexo de los hijos (Tabla 6), se encontraron diferencias significativas entre niños y niñas, respecto a la percepción de la frecuencia de expresiones de afecto y agresión de sus papás. Los niños perciben con mayor frecuencia dichas expresiones $(p<.01)$. Además, se encontró que los niños también perciben con mayor frecuencia expresiones de agresión de sus madres $(p<.000)$. 
Tabla 6. Expresiones de afecto-rechazo parental y sexo de los hijos.

\begin{tabular}{|c|c|c|c|c|c|c|c|c|c|c|}
\hline \multirow{3}{*}{ Expresiones } & \multicolumn{5}{|c|}{ Padres } & \multicolumn{5}{|c|}{ Madres } \\
\hline & \multicolumn{2}{|c|}{ Hijos } & \multicolumn{2}{|c|}{ Hijas } & \multirow[b]{2}{*}{$p$} & \multicolumn{2}{|c|}{ Hijos } & \multicolumn{2}{|c|}{ Hijas } & \multirow[b]{2}{*}{$p$} \\
\hline & $\mathrm{M}$ & $\mathrm{DE}$ & $\mathrm{M}$ & $\mathrm{DE}$ & & $\mathrm{M}$ & $\mathrm{DE}$ & $\mathrm{M}$ & $\mathrm{DE}$ & \\
\hline Afecto & 28.09 & 4.28 & 27.34 & 4.6 & $.009 * *$ & 28.59 & 3.31 & 28.07 & 4.52 & .972 \\
\hline Indiferencia & 9.91 & 3.27 & 9.56 & 3.55 & $.032 *$ & 11.53 & 2.25 & 11.37 & 2.51 & .053 \\
\hline Agresión & 9.33 & 3.22 & 8.4 & 2.76 & $.000 * *$ & 9.79 & 2.78 & 9.14 & 2.8 & $.000 * *$ \\
\hline Rechazo & 4.99 & 1.68 & 5.01 & 1.76 & .976 & 5.13 & 1.63 & 5.19 & 1.83 & .868 \\
\hline
\end{tabular}

* Diferencia significativa a nivel de $.05, * *$ Diferencia significativa a nivel de .01

Se evidenció que la frecuencia de las expresiones de afecto de las madres es diferente según la edad de los hijos ( $p=.0002)$, es mayor con los niños de 9 a 11 años, que con los de 12 a 14 años. No se encontró diferencia significativa en la frecuencia de las expresiones de afecto, indiferencia, agresión y rechazo de los papás y la edad de los hijos ( $p>.05)$, como se ve en la Tabla 7.

Tabla 7. Expresiones de afecto-rechazo parental y edad de los hijos.

\begin{tabular}{|c|c|c|c|c|c|c|c|c|c|c|}
\hline \multirow{3}{*}{ Expresiones } & \multicolumn{5}{|c|}{ Padres } & \multicolumn{5}{|c|}{ Madres } \\
\hline & \multicolumn{2}{|c|}{ De 9 a 11 años } & \multicolumn{2}{|c|}{ De 12 a 14 años } & \multirow[b]{2}{*}{$p$} & \multicolumn{2}{|c|}{ De 9 a 11 años } & \multicolumn{2}{|c|}{ De 12 a 14} & \multirow[b]{2}{*}{$p$} \\
\hline & $\mathrm{M}$ & $\overline{\mathrm{DE}}$ & $\mathrm{M}$ & $\mathrm{DE}$ & & $\mathrm{M}$ & $\mathrm{DE}$ & $\mathrm{M}$ & $\mathrm{DE}$ & \\
\hline Afecto & 27.8 & 4.3 & 26.7 & 5.8 & .274 & 28.5 & 3.8 & 26.9 & 4.8 & $.002 * *$ \\
\hline Indiferencia & 9.9 & 3.4 & 10.1 & 3.9 & .533 & 11.4 & 2.4 & 11.5 & 2.5 & .94 \\
\hline Agresión & 8.9 & 2.9 & 8.7 & 2.9 & .588 & 9.4 & 2.7 & 9.7 & 3.4 & .992 \\
\hline Rechazo & 4.9 & 1.6 & 5.3 & 2.4 & .503 & 5.1 & 1.7 & 5.6 & 2.3 & .252 \\
\hline
\end{tabular}

** Diferencia significativa a nivel de .01

Los resultados presentados en la Tabla 8 revelan que la frecuencia de las expresiones de agresión de los papás es diferente según el tipo de familia $(p=.016)$ y es mayor en las familias extendidas. También se encontró una diferencia significativa en la frecuencia de las expresiones de afecto de las madres, siendo más frecuentes en las familias nucleares $(p=.002)$.

Tabla 8. Expresiones de afecto-rechazo parental y tipo de familia.

\begin{tabular}{|c|c|c|c|c|c|c|c|c|c|c|}
\hline \multirow[b]{3}{*}{ Expresiones } & \multicolumn{5}{|c|}{ Padres } & \multicolumn{5}{|c|}{ Madres } \\
\hline & \multicolumn{4}{|c|}{ Tipo de familia } & \multirow[b]{2}{*}{$p$} & \multicolumn{4}{|c|}{ Tipo de familia } & \multirow[b]{2}{*}{$p$} \\
\hline & 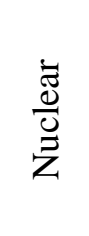 & 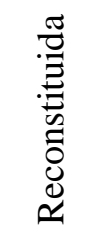 & 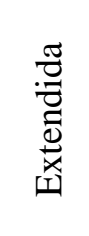 & 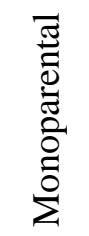 & & 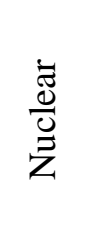 & 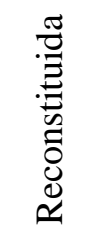 & 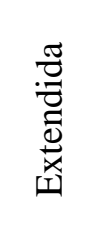 & 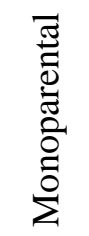 & \\
\hline Afecto & 28.0 & 25.7 & 27.5 & 27.3 & .142 & 28.8 & 26.2 & 27.9 & 28.3 & $.002 * *$ \\
\hline Indiferencia & 9.6 & 10 & 9.9 & 9.8 & .597 & 11.4 & 11.6 & 11.6 & 11.5 & .773 \\
\hline Agresión & 8.9 & 7.9 & 9.1 & 8.6 & .016 & 9.3 & 10.1 & 9.5 & 9.6 & .276 \\
\hline Rechazo & 4.9 & 5.6 & 4.9 & 5.1 & .497 & 5.1 & 6 & 4.9 & 5.3 & .151 \\
\hline
\end{tabular}

* Diferencia significativa a nivel de $.05, *$ Diferencia significativa a nivel de .01 


\section{DISCUSIÓN}

La investigación intenta definir la percepción de aceptación y rechazo que tienen los hijos sobre la conducta parental, considerando las características de los padres en cuanto a edad, sexo, nivel de instrucción y tipo de familia.

En los últimos años se considera más sistemáticamente el punto de vista de los hijos, realizándose investigaciones sobre manifestaciones de afecto o rechazo de sus padres y manejo de conflictos en el hogar (Oudhof van Barneveld et al., 2012; Richaud de Minzi et al., 2011; Rodríguez, 2017). Los resultados sugieren evaluar la conducta parental para obtener una mejor comprensión de la misma, no sólo en el modo en que informan de ella los padres o desde el recuerdo retrospectivo de los adultos, sino también a partir de la percepción de los hijos e hijas (Del Barrio et al., 2014). En la investigación de la conducta parental desde la percepción de los padres, en la que se utilizó el cuestionario PARQ Control (Clavijo et al., 2017), la mayoría de las preguntas evaluaban intenciones u opiniones de los padres sobre su conducta parental, los resultados obtenidos permitieron deducir que los padres contestan los ítems pensando en la educación de los hijos en general, pero no expresan el comportamiento real con los suyos (Solís-Cámara \& Díaz, 2007).

En cuanto a la percepción de aceptación y rechazo parental de los hijos, éstos perciben aceptación de sus padres y sus madres, datos que son coincidentes con estudios sobre percepciones de crianza en padres, madres e hijos (Del Barrio et al., 2014; Clavijo et al., 2017; Musitu \& Cava, 2003; Oudhof van Barneveld et al., 2012; Rodríguez, 2017). Actividades relacionadas con el interés, el apoyo, el afecto y la orientación, aparecen en un rango de siempre y frecuentemente, es decir, los padres y madres son considerados afectivos y apoyadores desde la percepción de su conducta parental. Resultados que coinciden con investigaciones efectuadas en la misma línea (Oudhof van Barneveld et al., 2012; Etxebarria et al., 2009; Del Barrio et al., 2014). De igual manera los datos obtenidos desde la percepción de los hijos son similares a los reportados por los padres (Clavijo et al., 2017). Derivaciones que permiten suponer que la ciudad de Cuenca no existe una visión radicalmente distinta entre padres e hijos a cerca de la percepción de aceptación y rechazo en las familias estudiadas.

En cuanto al sexo de los hijos se valoraron diferencias significativas entre la percepción de hijos e hijas en las dimensiones de aceptación y rechazo. Las hijas perciben como más afectivas a las madres que a los padres. En tanto que los hijos perciben en mayor frecuencia expresiones de agresión de sus papás. Esta situación puede explicarse posiblemente por la posición de la madre en la familia, puesto que a través del tiempo y en las diferentes culturas, la madre constituye la piedra angular en la crianza, siendo la figura con más cercanía con los hijos, generalmente comparte más tiempo con ellos, por lo que se constituye en la principal cuidadora (Oudhof van Barneveld et al., 2012; Etxebarria et al., 2009; Rodríguez, 2017). Hijos e hijas, tanto en la niñez como en la adolescencia, perciben a la madre más positivamente que al padre en lo que respecta a los aspectos emocionales y a los aspectos democráticos de la crianza. Las madres, a diferencia de los padres, son más aceptadas (Etxebarria et al., 2009) y son percibidas por los hijos como más comprensivas, más cercanas, más cariñosas, más implicadas en las tareas escolares de casa, con estilos de disciplina más flexibles e igualitarios, menos severas y más centradas en el hijo/a (Samper et al., 2006). En cambio, en poblaciones españolas con una muestra de adolescentes, los padres son percibidos como más autoritarios, rígidos, severos, restrictivos y formales (Rodríguez, 2009).

En relación con la instrucción de los padres, se encontró que los padres que registraron instrucción básica son percibidos con mayor frecuencia de agresión y rechazo. Estos resultados son similares a los obtenidos por Rodríguez (2017), quién sustenta la existencia de varios factores actitudinales y motivacionales, bienestar psicológico, momento de la paternidad, género, edad, temperamento, grado de formación y otros son específicos de la relación madre-padre que pueden incidir en las manifestaciones de afecto o rechazo de los padres con sus hijos. Al comparar las expresiones de aceptación y rechazo, según la edad de los padres, se encontró diferencias importantes en las manifestaciones de rechazo en padres menores de 38 años. Los niños participantes de esta investigación perciben a los padres menores de 38 años como más rechazadores. Según Etxebarria et al. (2009) esto puede darse porque a diferencia de otros tiempos, los padres jóvenes perciben la crianza como generadora de desacuerdos y conflictos con la pareja. Además, estos autores afirman que en la 
actualidad hombres y mujeres tienen una creciente preocupación por su carrera profesional y creen que tienen el derecho de anteponer la vida profesional a cualquier cosa: entre otras, al hecho de tener pareja, de tener hijos y cuidarlos. Estos aspectos pueden desencadenar los resultados obtenidos en las manifestaciones de afecto y rechazo desde la percepción de los hijos.

De igual manera la percepción de los hijos sobre las manifestaciones de afecto y rechazo parental se relaciona con la edad de los mismos. Estos resultados se confirman con la investigación de Etxebarria et al. (2009) quien señala que las expresiones de afecto de las madres son diferentes según la edad de los hijos. Otros estudios confirman que el grado de implicación de los padres con sus hijos cambia a medida que los niños crecen; por ejemplo, los padres se relacionan menos con sus hijos a medida que estos crecen y los hijos comienzan a relacionarse más con sus iguales, volviéndose limitada la influencia de sus padres (Samper et al., 2006). De igual manera se encuentra que los padres pasan más tiempo con los niños más pequeños que con los niños mayores (Rodríguez et al., 2017).

Los resultados en relación con el sexo de los padres y la percepción de las expresiones de afecto o rechazo por parte de los hijos indica que los niños de este estudio perciben a las madres como más afectivas y a los padres como exigentes. Estos hallazgos coinciden con la investigación de Oudhof van Barneveld et al. (2012) quien reporta un alto grado de coincidencia entre la percepción de crianza de los padres y de las madres, lo que se manifiesta en la consistencia en el ejercicio de parentalidad en cuanto a los niveles aceptación y rechazo impartido por los padres. De acuerdo con Rodríguez (2009), la mayor implicación y presencia de la mujer en la crianza explicaría la elevada aparición de interacciones positivas entre hijos y madres, en comparación con las interacciones que muestran los padres con los hijos, este investigador encontró que las madres se manifiestan más comunicativas, afectuosas, dialogantes, implicadas y preocupadas con sus hijos, teoría que permite sostener los resultados obtenidos.

Con relación a la percepción de los niños sobre las expresiones de afecto o rechazo parental, vinculado al tipo de familia, en este estudio se encontró que la agresión es mayor en familias extendidas, en tanto que las expresiones de afecto es mayor en madres de familias nucleares. Este resultado puede deberse a la influencia que ejercen los padres que conviven con sus hijos y los patrones de interacción que establecen con ellos al estar la presencia tanto del padre cuanto de la madre (Barneveld, Rodríguez, \& Robles, 2012; Rodríguez et al., 2017). Para Etxebarria et al. (2009) la relación armoniosa padremadre incrementa la probabilidad de la frecuencia y las interacciones positivas padre-niño en familias nucleares. Por el contrario, el conflicto marital, representa una barrera y es un predictor para la implicación del padre, lo que predice pobres resultados en los hijos, situación que suele darse en caso de las familias extendidas, puesto que puede presentarse cierta confusión respecto a la posición y el papel de los padres respecto a los hijos, o la falta de tiempo para la implicación parental en la crianza de los hijos.

\section{CONCLUSIONES}

Los resultados confirman que existe una tendencia positiva en las manifestaciones de afecto parental. Desde la percepción de los hijos, padres y madres son afectuosos, sin embargo, tanto niños, como las niñas coinciden en ver a la madre como más afectuosa que el padre, aunque también, para los hijos en esta investigación, es la madre la que manifiesta expresiones de indiferencia y agresión con mayor frecuencia. De igual manera, los resultados obtenidos en este estudio permiten concluir que la percepción de manifestaciones, tanto de aceptación cuanto de rechazo, están vinculadas con el nivel de instrucción de los padres, a la percepción de los hijos se encuentra mayores manifestaciones de agresión y rechazo en padres que tienen instrucción básica, siendo los resultados menores en padres con bachillerato o instrucción superior. Respecto a la edad de los padres, se encontraron diferencias significativas en las manifestaciones de rechazo, siendo mayor en progenitores menores a los 38 años. En relación con el tipo de familia se concluye que los niños que viven en familias nucleares tienden a percibir una mayor aceptación de los padres, por cuanto los dos están involucrados y comprometidos en sus vidas. Es prudente pensar que los padres de familias nucleares tienen mayores oportunidades para mostrar su apoyo e involucrarse en la vida cotidiana de sus hijos, por ejemplo, hablar con ellos, 
facilitar apoyo emocional, ayudar con las tareas y los problemas habituales, establecer normas o vigilar el comportamiento de los hijos, por lo que estas condiciones podrían explicar la percepción del apoyo y el afecto de los padres de este tipo de familia.

\section{Limitaciones}

En general se debe considerar la escasez de estudios sobre percepción de la conducta parental en niños pequeños, particularmente con muestras cuencanas y ecuatorianas, no se cuenta con evidencia consistente que permita comprender los efectos de la percepción de aceptación y rechazo parental en la conducta de los hijos. Al trabajar con una muestra intencional en una ciudad pequeña, que incluyó tipología de familias diversas, hace que los resultados obtenidos no puedan generalizarse a otras poblaciones. A más de explorar la valoración que tienen los hijos analizando en qué grado muestran aceptación o rechazo los padres hacia ellos, el presente trabajo podría corroborarse con un estudio que mida las frecuencias con las que se realizan esas relaciones. Finalmente, se evidencia la necesidad de desarrollar más investigaciones que evalúen el grado en que las percepciones de afecto o rechazo parental de los hijos puede ser conceptualizada de forma similar o diferente para los padres y las madres y por los hijos en distintas edades. Posiblemente quedan por identificar variables como el contexto psicosocial, la edad y sexo del niño, la estructura familiar, la significación clínica del ajuste del niño o el estatus económico o profesional de los padres, entre otros, que podrían introducir modificaciones en los patrones del comportamiento de los padres en la crianza y por tanto en la percepción de los hijos.

\section{REFERENCIAS}

Álvarez, M. (2010). Prácticas educativas parentales: autoridad familiar, incidencia en el comportamiento agresivo infantil. Revista Virtual Universidad Católica del Norte, 31, 253-273.

Caycho, T. P. (2016). Relación con los padres y estrategias de afrontamiento en adolescentes de Lima. Propósitos y Representaciones, 4(1), 11-59. http://dx.doi.org/10.20511/ pyr2016.v4n1.86

Clavijo, R., Palacios, M., Mora, C., Villavicencio, F., Arpi, N., Conforme, G. (2017). Aceptación rechazo de padres y madres cuencanos relacionados a las características personales de los hijos entre 5 y 12 años. Revista Maskana, Vol. 7(2017), Número Especial: Actas del Congreso de Neurociencias.

Del Barrio, V., Ramírez-Uclés, I., Romero, C., Carrasco, M. A. (2014). Adaptación del ChildPARQ/Control: versiones para el padre y la madre en población infantil y adolescente española. Acción Psicológica, 11(2), 27-46. http://dx.doi.org/10.5944/ap.11.2.14173

Evans, J., Myers, R. (1994). Prácticas de crianza: Creando programas donde las tradiciones y las prácticas modernas se encuentran. Childrearing, 15, 1-11.

Espinal, I., Gimeno, A., González, F. (2006). El enfoque sistémico en los estudios sobre la familia. Revista internacional de sistemas, 14, 21-34.

Etxebarria, I., Apodaca, P., Fuentes, M., López, F., Ortiz, M. J. (2009). La crianza y la educación de los hijos en la sociedad actual: ¿lo estamos haciendo bien? Revista de Psicología Social, 24(1), 81-96, http://dx.doi.org/10.1174/021347409786922952

Gaylord, N. K., Kitzmann, K. M., Coleman, J. K. (2003). Parents' and children's perceptions of parental behaviour: Associations with children's psychosocial adjustment in the classroom. Parenting, 3, 23-47. http://dx.doi.org/10.1207/s15327922par0301_02

Gracia, E., Lila, M., Musitu, G. (2005). Rechazo parental y ajuste psicológico y social de los hijos. Revista Salud Mental, 28(2), 73-81.

Musitu, G., Cava, M. (2001). La familia y la educación. Biblioteca Latinoamericana de Educación, $223 \mathrm{p}$. 
Oudhof van Barneveld, H., Rodríguez, B., Robles É. (2012). La percepción de la crianza en padres, madres e hijos adolescentes pertenecientes al mismo núcleo familiar. Liberabit, 18(1), 75-82.

Polo del Río, M., Fajardo, F., Martín, E., Gómez, T., León, B. (2012). Aceptación/rechazo parental en una muestra de adolescentes. Diferencias según sexo y edad. INFAD Revista de Psicología, 1(1), 329-336.

Richaud de Minzi, M., Lemos, V., Mesurado, B. (2011). Relaciones entre la percepción que tienen los niños de los estilos de relación y de la empatía de los padres y la conducta prosocial en la niñez media y tardía. Avances en Psicología Latinoamericana, 29(2), 330-343.

Rodríguez, M., Del Barrio, M., Carrasco, M. (2009). ¿Cómo perciben los hijos la crianza materna y paterna? Diferencias por edad y sexo. Escritos de Psicología, 2(2), 10-18.

Rodríguez, M. (2017). Análisis de la implicación del padre en el ajuste psicológico: Mediación de la aceptación- rechazo parental percibida. Universidad Nacional de Educación a Distancia (España). Facultad de Psicología. Departamento de la Personalidad, Evaluación y Tratamiento Psicológicos. Tesis de grado. Disponible en http://espacio.uned.es/fez/view/tesisuned:Psicologia-Mmrodriguez

Rohner, R., Carrasco, M. (2014). Teoría de la Aceptación Rechazo Interpersonal (IPARTheory): Bases Conceptuales, método y evidencia empírica. Acción Psicológica, 11(2), 9-25.

Samper, P., Cortés, M., Mestre, V., Nacher, M., Tur, A. (2006). Adaptación del Childs Report of Parent Behavior Inventory a población española. Psicothema, 18(2), 263-271.

Solís-Cámara, P., Díaz, M. (2007). Relaciones entre creencias y prácticas de crianza de padres con niños pequeños. Anales de Psicología, 23(2), 177-184.

Torres, L. E., Ortega, P., Garrido, A., Luna, A. (2008). Dinámica familiar en familias con hijos e hijas. Revista Intercontinental de Psicología y Educación, 10(2), 31-56.

Triana, A., Ávila, L., Malagón, A. (2010). Patrones de crianza y cuidado de niños y niñas en Boyacá. Revista Latinoamericana, Ciencias Sociales, Niñez y Juventud, 8(2), 933-945. 